\title{
Extreme rays in $3 \otimes 3$ entangled edge states with positive partial transposes
}

\author{
Woo Chan Kim ${ }^{a}$, \\ ${ }^{a}$ Department of Mathematics, Seoul National University, Seoul 151-742, KOREA \\ Seung-Hyeok Kye ${ }^{\text {b,1, }}$ \\ ${ }^{\mathrm{b}}$ Department of Mathematics, Seoul National University, Seoul 151-742, KOREA
}

\begin{abstract}
In this paper, we show that the $3 \otimes 3$ edge PPTES of the type $(5,5)$ found by Clarisse and Ha generate extreme rays in the cone of all positive semi-definite matrices with positive semi-definite partial transposes.
\end{abstract}

Key words: entangled edge state, positive partial transpose, extreme ray PACS: 03.65.Bz, 03.67.-a, 03.67.Hk

1991 MSC: 81P15, 46L05, 15A30

\section{Introduction}

The notion of entanglement in quantum physics has been studied extensively by physicists during the last decade in connection with the quantum information theory and quantum communication theory. One of the basic questions in the theory of entanglement is how to determine if a given state is entangled or not.

A density matrix $A$ in $\left(M_{n} \otimes M_{m}\right)^{+}$is said to be entangled if it does not belong to $M_{n}^{+} \otimes M_{m}^{+}$, where $M_{n}^{+}$denotes the cone of all positive semi-definite

Email addresses: wckim@math.snu.ac.kr (Woo Chan Kim), kye@snu.ac.kr (Seung-Hyeok Kye).

1 This work was partially supported by KRF grant (R14-2003-006-01002-0). 
$n \times n$ matrices over the complex fields. A density matrix is said to be separable if it belongs to $M_{n}^{+} \otimes M_{m}^{+}$. Recall that a density matrix defines a state on the matrix algebra by the Schur or Hadamard product.

In the early eighties, it was observed by Choi [1] that the partial transpose of every separable state is positive semi-definite. The partial transpose or block transpose $A^{\tau}$ of $A \in M_{n} \otimes M_{m}$ is defined by

$$
\left(\sum_{i, j=1}^{m} a_{i j} \otimes e_{i j}\right)^{\tau}=\sum_{i, j=1}^{m} a_{j i} \otimes e_{i j} .
$$

This necessary condition for separability has been rediscovered by Peres [10], and is called the PPT criterion for separability. Choi [1] also gave an example of $3 \otimes 3$ entangled state whose partial transpose is positive semi-definite. This kind of entangled state is called PPTES. There are many examples of $3 \otimes 3$ PPTES in the literature. See the references in [5].

In this note, we denote by $\mathbb{T}$ the convex cone of all positive semi-definte block matrices whose partial transposes are also positive semi-definite. In other words,

$$
\mathbb{T}=\left\{A \in\left(M_{n} \otimes M_{m}\right)^{+}: A^{\tau} \in\left(M_{n} \otimes M_{m}\right)^{+}\right\} .
$$

In order to understand PPTES, it seems to be essential to know the facial structures of the cone $\mathbb{T}$. In this sense, the notion of edge PPTES plays an important role as was introduced in [9]. A PPTES $A$ in $\mathbb{T}$ is an edge PPTES if and only if the proper face of $\mathbb{T}$ containing $A$ as an interior point does not contain a separable state.

Edge PPTES may be classified by their range dimensions as was studied in [11]. An edge PPTES $A$ is said to be of the type $(s, t)$ if the range dimension of $A$ is $s$, and the range dimension of $A^{\tau}$ is $t$. There are many examples of $(4,4)$ and $(6,7)$ edge PPTES in the literature, and PPTES of the types $(7,5)$, $(6,5)$ and $(8,5)$ were found by the second author in [5]. Very recently, $(5,5)$ and $(6,6)$ edge PPTES were also found by Clarisse [2] and Ha [4].

In [11], the authors conjectured that every $3 \otimes 3$ PPTES has the Schmidt numbers 2, and showed that this is the case for $(4,4)$ edge PPTES. By the duality theory [3] between positive linear maps and entanglement, this conjecture is equivalent to asking whether every 2-positive linear map in $M_{3}$ is decomposable, which was also studied by operator algebraists. 
One of the key steps to understand a given convex cone is to find all extreme rays. We say that a PPTES is extremal if it generates an extreme ray in the convex cone $\mathbb{T}$. One of possible approach to the above conjecture is to show that every extremal PPTES is of the type $(4,4)$. At a glance, this approach seems to be quite reasonable since 4 is the smallest range dimension of edge PPTES by an argument in [8]. The second author [7] also showed that a family of $(4,4)$ PPTES are extremal by a direct argument.

The purpose of the present paper is to show that the above mentioned PPTES of the type $(5,5)$ by Clarisse and Ha are also extremal. These constitute the first known examples of extremal PPTES which are not of the type $(4,4)$. We explain very briefly in the next section the basic strategy for proofs in connection with the facial structures of the cone $\mathbb{T}$, and show the extremality of Clarisse and Ha's examples in the last section.

Throughout this paper, we will not use bra-ket notations. Every vector will be considered as a column vector. If $x \in \mathbb{C}^{m}$ and $y \in \mathbb{C}^{n}$ then $x$ will be considered as an $m \times 1$ matrix, and $y^{*}$ will be considered as a $1 \times n$ matrix, and so $x y^{*}$ is an $m \times n$ rank one matrix whose range is generated by $x$ and whose kernel is orthogonal to $y$. The notation $(\cdot \mid \cdot)$ will be used for the inner product, which is sesqui-linear, that is, linear in the first variable and conjugate-linear in the second variable. For natural numbers $m$ and $n$, we denote by $m \vee n$ and $m \wedge n$ the maximum and minimum of $m$ and $n$, respectively.

\section{Facial structures for PPTES}

For an $m \times n$ matrix $z=\left[z_{i k}\right] \in M_{m \times n}$, we define

$$
\begin{aligned}
& z_{i}=\sum_{k=1}^{n} z_{i k} e_{k} \in \mathbb{C}^{n}, \quad i=1,2, \ldots, m, \\
& \widetilde{z}=\sum_{i=1}^{m} z_{i} \otimes e_{i} \in \mathbb{C}^{n} \otimes \mathbb{C}^{m} .
\end{aligned}
$$

Then $z \mapsto \widetilde{z}$ defines an inner product isomorphism from $M_{m \times n}$ onto $\mathbb{C}^{n} \otimes \mathbb{C}^{m}$. We also note that $\widetilde{z} \widetilde{z}^{*}$ is a positive semi-definite matrix in $M_{n} \otimes M_{m}$ of rank 
one. We consider the convex cones

$$
\begin{aligned}
\mathbb{V}_{s} & =\operatorname{conv}\left\{\widetilde{z} \widetilde{z}^{*}: \operatorname{rank} z \leq s\right\}, \\
\mathbb{V}^{s} & =\operatorname{conv}\left\{\left(\widetilde{z} \widetilde{z}^{*}\right)^{\tau}: \operatorname{rank} z \leq s\right\} .
\end{aligned}
$$

for $s=1,2, \ldots, m \wedge n$. A density matrix in $M_{n} \otimes M_{m}$ is separable if and only if it belongs to the cone $\mathbb{V}_{1}$ as was explained in [5]. We also, note that

$$
\mathbb{T}=\mathbb{V}_{m \wedge n} \cap \mathbb{V}^{m \wedge n}
$$

It is well known that every face of $\mathbb{V}_{m \wedge n}=\left(M_{n} \otimes M_{m}\right)^{+}$and $\mathbb{V}^{m \wedge n}$ is of the form

$$
\begin{aligned}
& \Psi_{D}=\left\{A \in\left(M_{n} \otimes M_{m}\right)^{+}: \mathcal{R} A \subset \widetilde{D}\right\}, \\
& \Psi^{E}=\left\{A \in M_{n} \otimes M_{m}: A^{\tau} \in \Psi_{E}\right\},
\end{aligned}
$$

respectively, where $\mathcal{R} A$ is the range space of $A$ and $\widetilde{D}=\left\{\widetilde{z} \in \mathbb{C}^{n} \otimes \mathbb{C}^{m}: z \in D\right\}$ for the subspace $D \subset M_{m \times n}$. It is also easy to see that every face of $\mathbb{T}$ is of the form

$$
\tau(D, E):=\Psi_{D} \cap \Psi^{E}
$$

for a pair $(D, E)$ of subspaces of $M_{m \times n}$, as was explained in [6]. The set $\tau(D, E)$ is a face of $\mathbb{T}$ if and only if there exist $A \in\left(M_{n} \otimes M_{m}\right)^{+}$such that

$$
\mathcal{R} A=\widetilde{D}, \quad \mathcal{R} A^{\tau}=\widetilde{E}
$$

The interior of the face $\tau(D, E)$ is given by

$$
\operatorname{int} \tau(D, E)=\left\{A \in\left(M_{n} \otimes M_{m}\right)^{+}: \mathcal{R} A=\widetilde{D}, \mathcal{R} A^{\tau}=\widetilde{E}\right\}
$$

Let $A$ be a PPTES. Then there is a unique face $\tau(D, E)$ in which $A$ is an interior point. In order to show that $A$ is extremal, it is enough to show that every member in $\tau(D, E)$ is a scalar multiple of $A$. We will follow the following steps to show that $A$ is extremal.

Step 1: Find a basis of the space $D$, and describe the general form of matrices in $\Psi_{D}$. We note by $A_{1}$ this general form, and impose the condition that $A_{1}^{\tau}$ is positive semi-definite to get a simpler form.

Step 2: Do the same thing for the space $E$, and get a simple form $A_{2}$ for matrices in $\Psi^{E}$.

Step 3: Compare $(i, j)$ entries of $A_{1}$ and $A_{2}$ to show that they reduce to scalar multiples of $A$. 


\section{$3 \quad$ Examples}

We begin with the example of Ha in [4]. His example has two parameter, and is given by

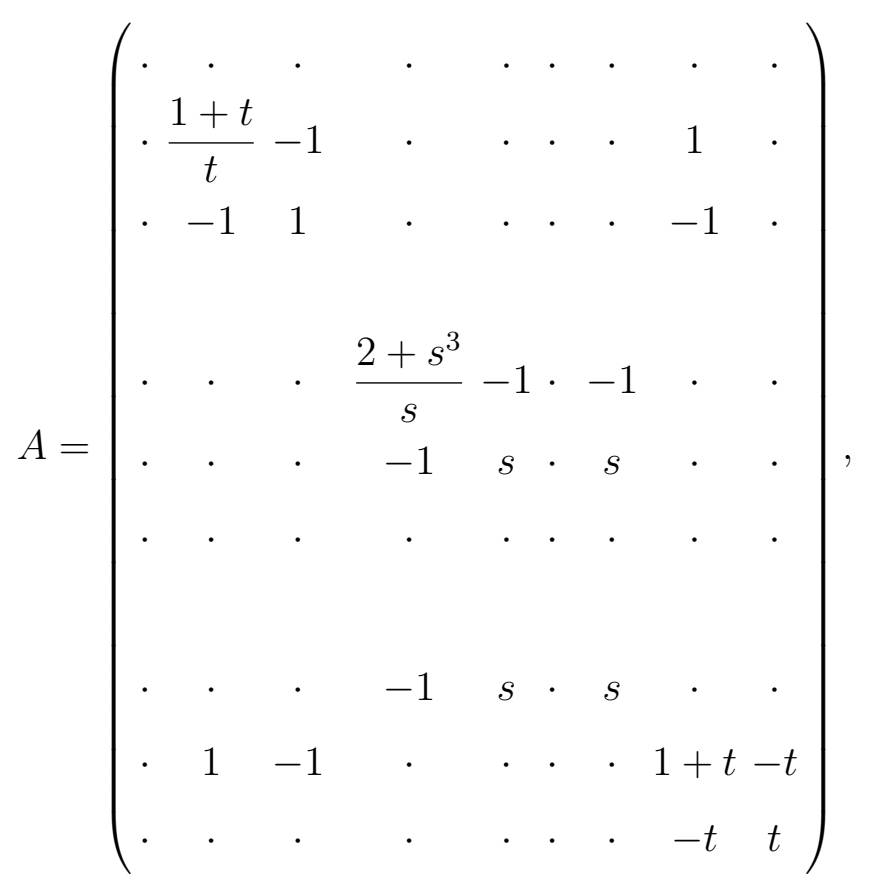

where $s, t>0$, and $\cdot$ denotes 0 . We choose

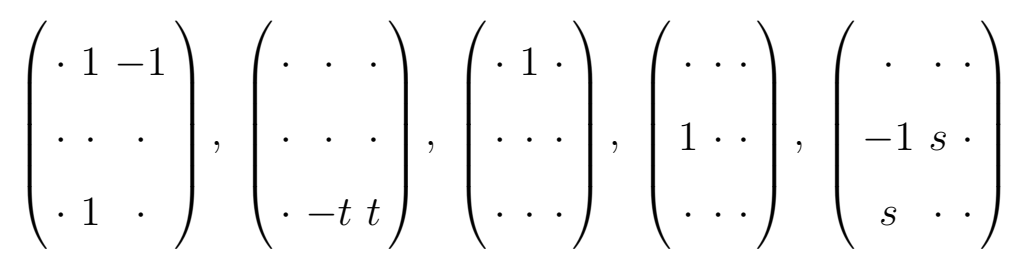

for a basis of $D$. Then every matrix $x_{i} \in D$ is of the form

$$
x_{i}=\left(\begin{array}{ccc} 
& \xi_{i}+\zeta_{i} & -\xi_{i} \\
\lambda_{i}-\mu_{i} & s \mu_{i} & \cdot \\
s \mu_{i} & \xi_{i}-t \eta_{i} & t \eta_{i}
\end{array}\right)
$$


So, $A_{1}=\sum \widetilde{x_{i}} \widetilde{x_{i}}{ }^{*} \in \Psi_{D}$ is of the form

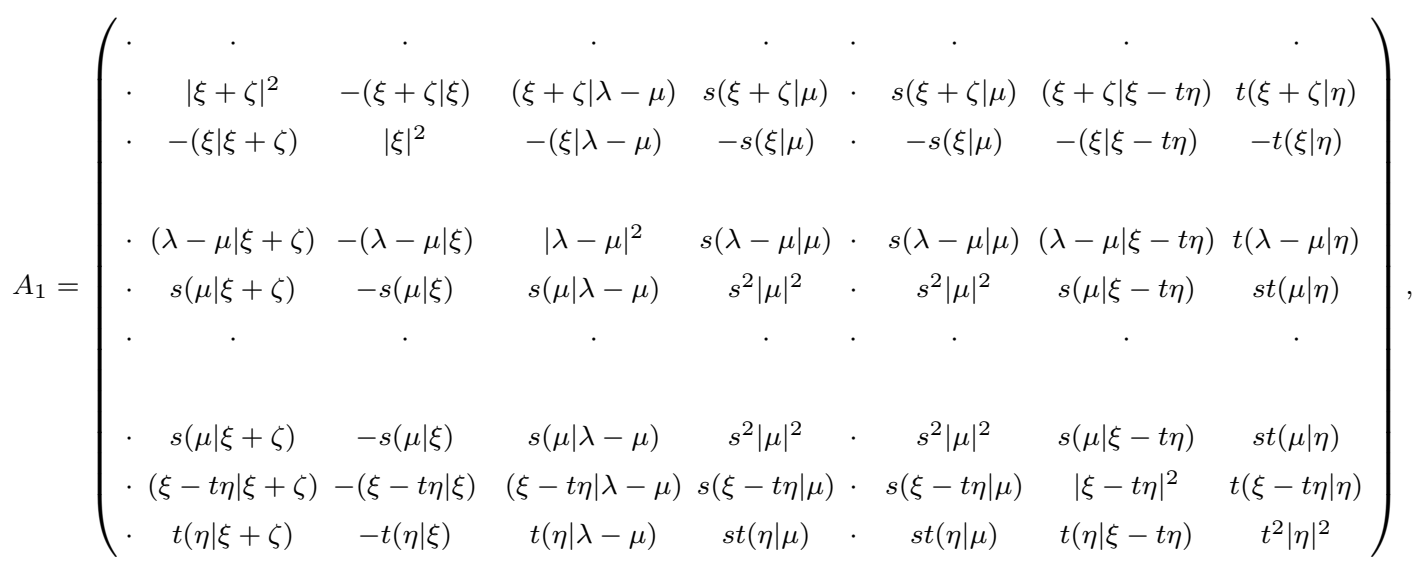

if we denote by $\xi, \eta, \zeta, \lambda$ and $\mu$ the vectors whose entries are $\xi_{i}, \eta_{i}, \zeta_{i}, \lambda_{i}$ and $\mu_{i}$, respectively. So, $A_{1}^{\tau}$ is of the form

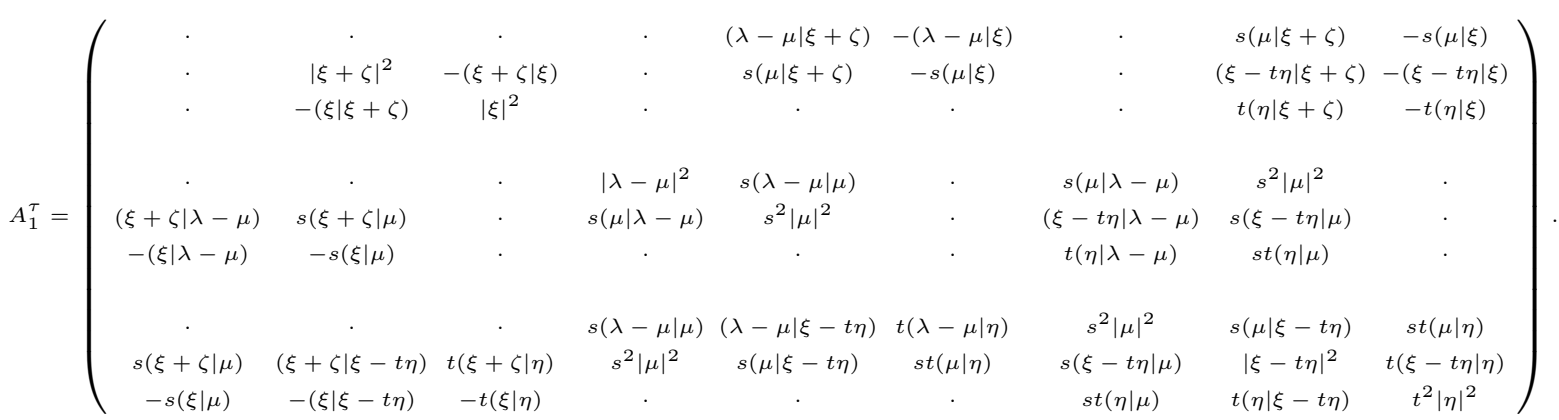

Now, we consider the condition that $A_{1}^{\tau}$ is positive semi-definite. We consider the following combinations

$$
(1,5),(1,8),(1,9),(1,6),(7,6),(8,6)
$$

of columns and rows to extract $2 \times 2$ submatrices of $A_{1}^{\tau}$, and see that $\{\xi, \eta, \zeta\}$ and $\{\lambda, \mu\}$ are orthogonal each other. This gives us the following simpler form of $A_{1}$ :

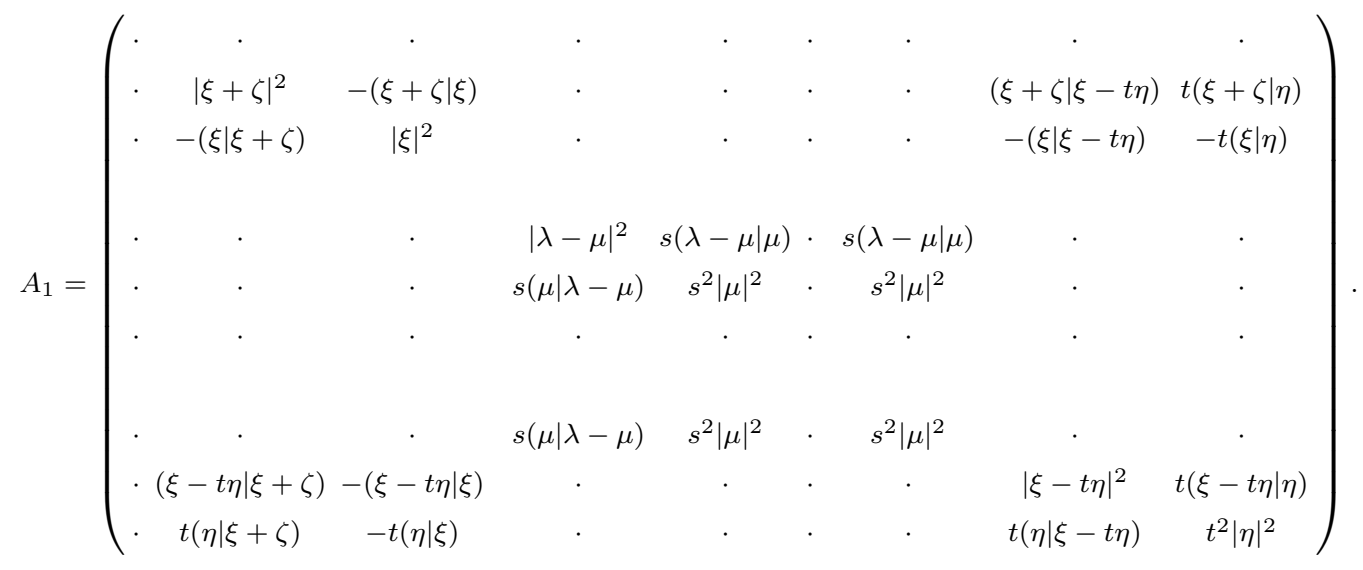


For the second step, we choose a basis

$$
\left(\begin{array}{c}
\cdots \\
s \cdot \\
\cdot 1 \cdot
\end{array}\right),\left(\begin{array}{cc}
\cdot & . \\
-1 & s \cdot \\
\cdot & .
\end{array}\right),\left(\begin{array}{cc}
\cdot & \cdots \\
-1 & \cdots \\
s & \cdots
\end{array}\right),\left(\begin{array}{c}
\cdot 1 \cdot \\
\ldots \\
\cdot t
\end{array}\right),\left(\begin{array}{ccc}
\cdot & -1 & 1 \\
\cdot & \cdot & \cdot \\
\cdot & \cdot & \cdot
\end{array}\right)
$$

of the space $E$. Then, every $y_{i} \in E$ is of the form

$$
y_{i}=\left(\begin{array}{ccc}
\cdot & \delta_{i}-\epsilon_{i} & \epsilon_{i} \\
s \alpha_{i}-\beta_{i}-\gamma_{i} & s \beta_{i} & \cdot \\
s \gamma_{i} & \alpha_{i}+t \delta_{i} & -t \delta_{i}
\end{array}\right) .
$$

¿From this, we construct $A_{2}^{\tau}=\sum \widetilde{y}_{i} \widetilde{y}_{i}{ }^{*} \in \Psi_{E}$. We denote by $\alpha, \beta, \gamma, \delta$ and $\epsilon$ the vectors whose entries are $\alpha_{i}, \beta_{i} \gamma_{i}, \delta_{i}$ and $\epsilon_{i}$, respectively, and put $\omega=$ $s \alpha-\beta-\gamma$. By the similar argument as above, we see $\{\alpha, \beta, \gamma\}$ and $\{\lambda, \mu\}$ are orthogonal each other, and get for following form for $A_{2}$ :

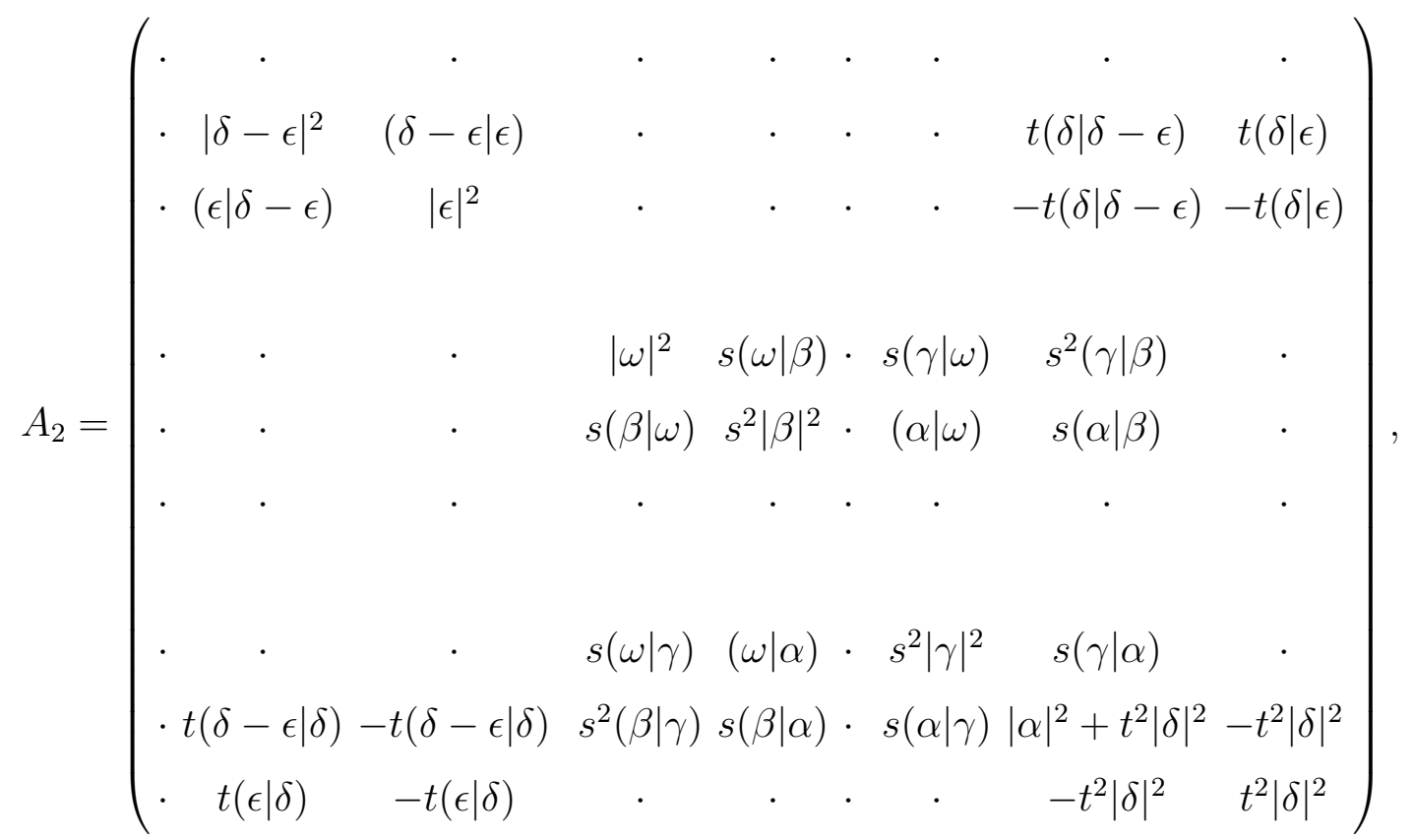

Now, we compare entries of $A_{1}$ and $A_{2}$. First, we compare both of $(2,9)$ and $(3,9)$ entries to see that $\eta \perp \zeta$. Similarly, we get $\xi \perp \zeta$ from the $(2,8)$ and $(3,8)$ entries, and $\xi \perp \eta$ from $(8,9)$ and $(9,9)$ entries. If we compare both $(4,5)$ and $(5,5)$ entries then we also get $\lambda \perp \mu$, since $\omega=s \alpha-\beta-\gamma$. Therefore, we see that the following families

$$
\xi, \eta, \zeta, \lambda, \mu
$$


are orthogonal each other. Next, we compare $(4,8),(5,8)$ and $(7,8)$ entries of $A_{1}$ and $A_{2}$, to see that $\alpha, \beta, \gamma$ are orthogonal each other. Finally, we see that $\delta \perp \epsilon$ to compare $(3,9)$ entries, since $\xi \perp \eta$. Therefore, we conclude that

$$
\alpha, \beta, \gamma, \delta, \epsilon
$$

are orthogonal each other. We put these conditions together to get the following forms for $A_{1}$ and $A_{2}$ as follows:

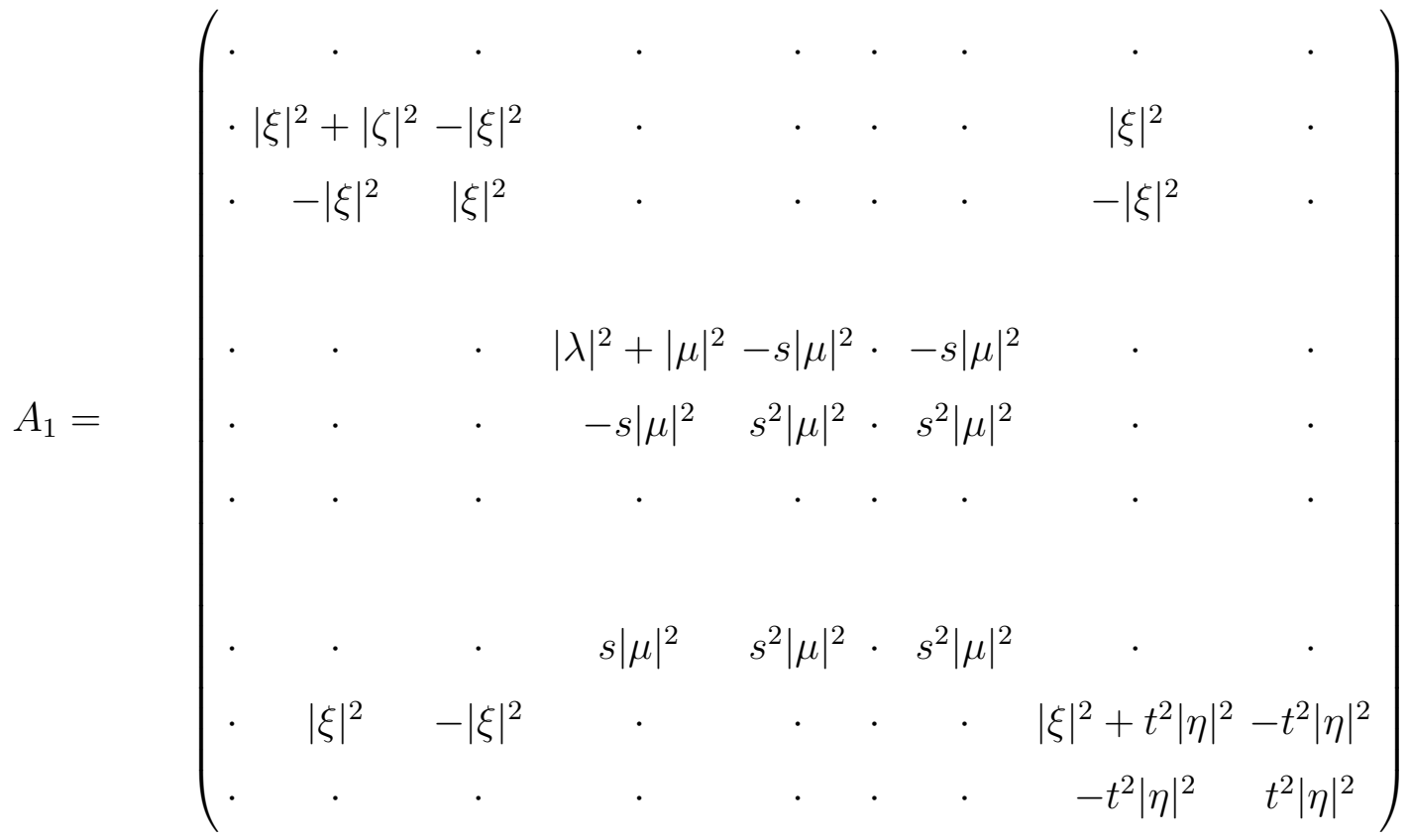

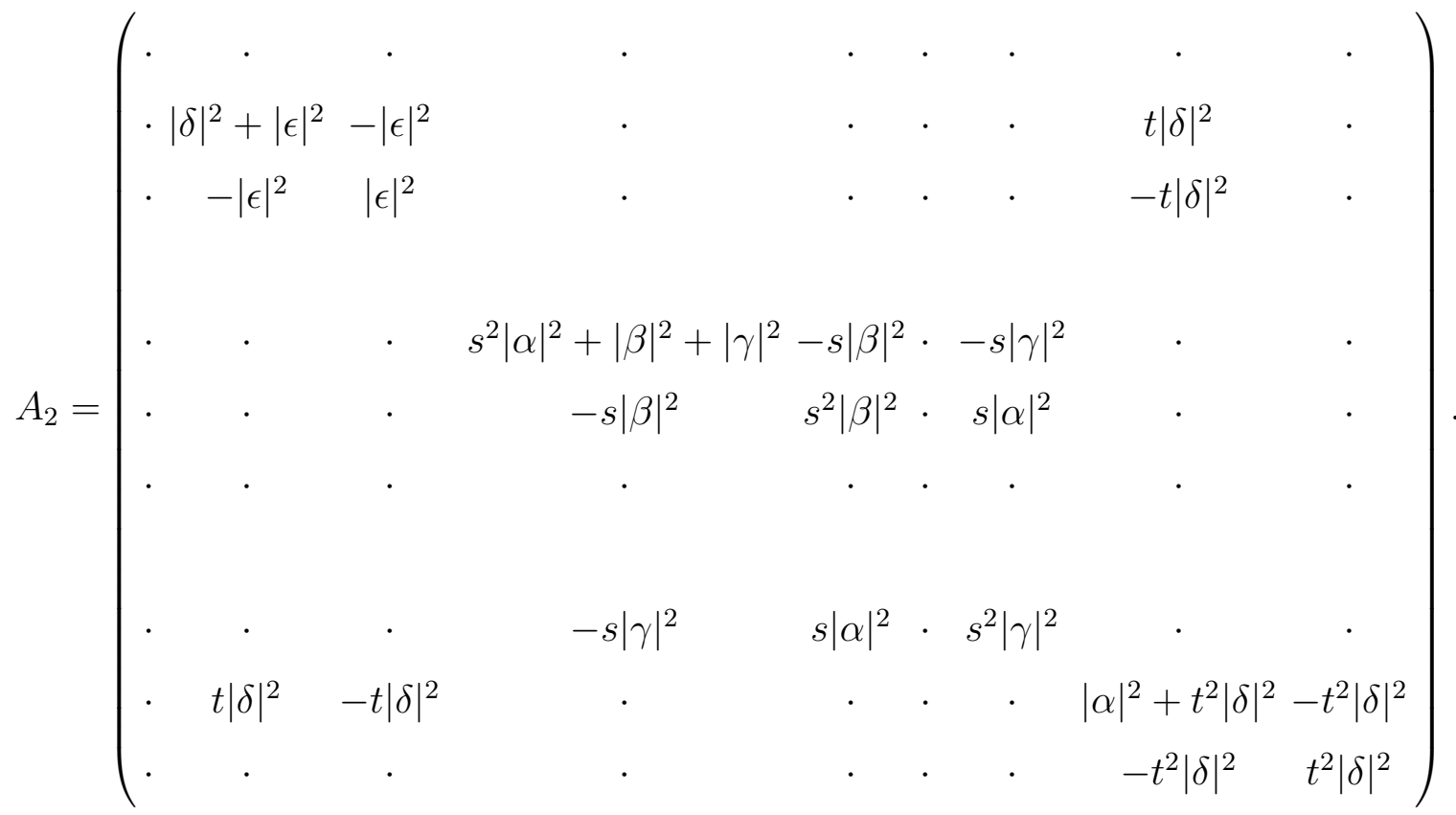


Finally, we compare $(5,5),(7,5)$ and $(7,7)$ entries of $A_{1}$ and $A_{2}$, to see that

$$
\frac{1}{s}|\alpha|^{2}=|\beta|^{2}=|\gamma|^{2}
$$

We also compare $(2,3)$ and $(2,8)$ entries, to get

$$
t|\delta|^{2}=|\epsilon|^{2}
$$

We note that $(8,8)$ entry of $A_{1}$ equals to the sum of $(3,3)$ and $(9,9)$ entries. So, we have

$$
|\alpha|^{2}+t^{2}|\delta|^{2}=|\epsilon|^{2}+t^{2}|\delta|^{2}
$$

in $A_{2}$, and it follows that

$$
|\alpha|^{2}=|\epsilon|^{2}
$$

Therefore, if we put $|\alpha|^{2}=1$ then we have

$$
|\beta|^{2}=|\gamma|^{2}=\frac{1}{s}, \quad|\delta|^{2}=\frac{1}{t}, \quad|\epsilon|^{2}=1
$$

This means that every matrix in $\tau(D, E)$ is the scalar multiple of the matrix $A$, and completes the proof that $A$ generates an extreme ray in the cone $\mathbb{T}$.

The example of Clarisse in [2] is given by

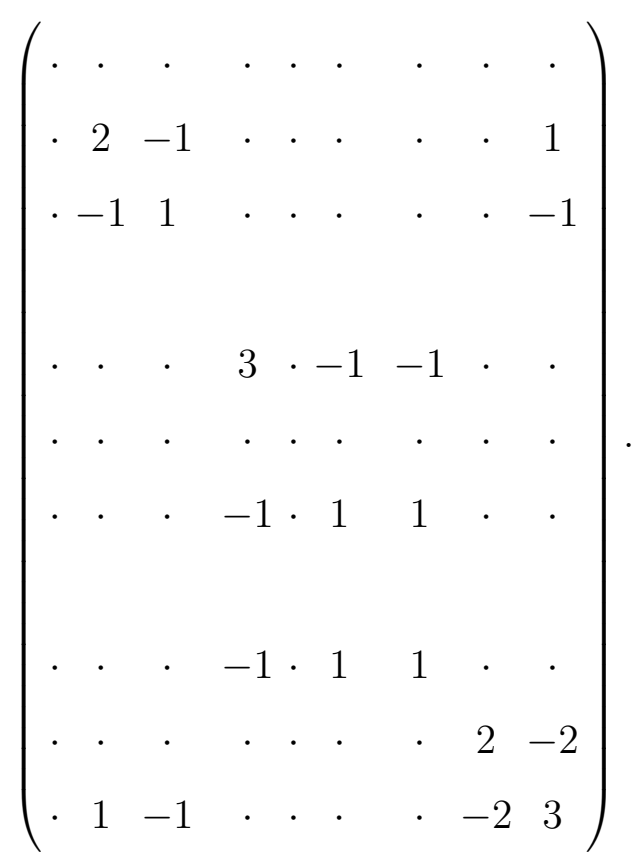


In this case, we choose a basis

$$
\left(\begin{array}{l}
\cdot 1 \cdot \\
\cdots \\
\cdots
\end{array}\right),\left(\begin{array}{ll}
\cdots & -1 \\
\cdots & \cdot \\
\cdots & 1
\end{array}\right),\left(\begin{array}{ll}
\cdots \\
\cdots \\
\cdot 1 & -1
\end{array}\right),\left(\begin{array}{l}
\cdots \\
1 \cdots \\
\cdots
\end{array}\right),\left(\begin{array}{c}
\cdot \cdot \\
\cdot \cdot-1 \\
-1 \cdot
\end{array}\right)
$$

of $D$, and a basis of

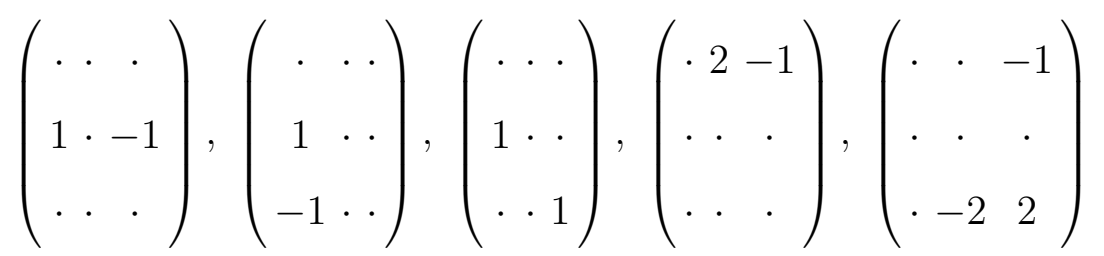

of $E$. Then the exactly same argument shows that the example of Clarisse is extremal.

We have tried to prove that examples of $(6,6)$ edge PPTES by Clarisse and $\mathrm{Ha}$ are extremal by the same argument. But, we had difficulty to apply our method in these cases, since the matrices in the Step 1 are much more complicated.

The authors are grateful to the referee for valuable comments.

\section{References}

[1] M.-D. Choi, Positive linear maps, Operator Algebras and Applications (Kingston, 1980), pp. 583-590, Proc. Sympos. Pure Math. Vol 38. Part 2, Amer. Math. Soc., 1982.

[2] L. Clarisse, Construction of bound entangled edge states with special ranks, Phys. Lett. A 359 (2006) 603.

[3] M.-H. Eom and S.-H. Kye, Duality for positive linear maps in matrix algebras, Math. Scand. 86 (2000), 130-142.

[4] K.-C. Ha, Comment on : "Construction of bound entangled edge states with special ranks" [Phys. Lett. A 359 (2006) 603], Phys. Lett. A 361 (2007) 515519.

[5] K.-C. Ha, S.-H. Kye, Construction of $3 \otimes 3$ entangled edge states with positive partial transposes, J. Phys. A 38 (2005), no. 41, 9039-9050.

[6] K.-C. Ha and S.-H. Kye, Construction of entangled states with positive partial transposes based on indecomposable positive linear maps, Phys. Lett. A $\mathbf{3 2 5}$ (2004), 315-323. 
[7] K.-C. Ha, S.-H. Kye and Y. S. Park, Entanglements with positive partial transposes arising from indecomposable positive linear maps, Phys. Lett. A 313 (2003), 163-174.

[8] P. Horodecki, M. Lewenstein, G. Vidal and I. Cirac, Operational criterion and constructive checks for the separablity of low rank density matrices, Phys. Rev. A 62 (2000), 032310.

[9] M. Lewenstein, B. Kraus, J. I. Cirac and P. Horodecki, Optimization of entanglement witnesses, Phys. Rev. A 62 (2000), 052310.

[10] A. Peres, Separability criterion for density matrices, Phys. Rev. Lett. 77 (1996), 1413-1415.

[11] A. Sanpera, D. Bruß and M. Lewenstein, Schmidt number witnesses and bound entanglement, Phys. Rev. A 63 (2001), 050301. 